University of Nebraska - Lincoln

DigitalCommons@University of Nebraska - Lincoln

1999

\title{
Routing and Wavelength Assignment (RWA) with Power Considerations In All- Optical Wavelength-Routed Networks
}

\author{
Maher Ali \\ University of Nebraska-Lincoln \\ Byrav Ramamurthy \\ University of Nebraska-Lincoln, bramamurthy2@unl.edu \\ Jitender S. Deogun \\ University of Nebraska-Lincoln, jdeogun1@unl.edu
}

Follow this and additional works at: https://digitalcommons.unl.edu/cseconfwork

Part of the Computer Sciences Commons

Ali, Maher; Ramamurthy, Byrav; and Deogun, Jitender S., "Routing and Wavelength Assignment (RWA) with Power Considerations In All- Optical Wavelength-Routed Networks" (1999). CSE Conference and Workshop Papers. 60.

https://digitalcommons.unl.edu/cseconfwork/60

This Article is brought to you for free and open access by the Computer Science and Engineering, Department of at DigitalCommons@University of Nebraska - Lincoln. It has been accepted for inclusion in CSE Conference and Workshop Papers by an authorized administrator of DigitalCommons@University of Nebraska - Lincoln. 


\title{
Routing and Wavelength Assignment (RWA) with Power Considerations In All- Optical Wavelength-Routed Networks
}

\author{
Maher Ali, Byrav Ramamurthy, and Jitender S. Deogun \\ Department of Computer Science \& Engineering \\ University of Nebraska-Lincoln \\ Lincoln, NE 68588-0115 U.S.A.
}

\begin{abstract}
Routing and wavelength assignment (RWA) is an important problem that arises in wavelength division multiplexed (WDM) optical networks. Previous studies have solved many variations of this problem under the assumption of perfect conditions regarding the power of a signal. In this paper, we investigate this problem while allowing for degradation of routed signals by components such as taps, multiplexers, and fiber links. We assume that optical amplifiers are preplaced. We investigate the problem of routing the maximum number of connections while maintaining proper power levels. The problem is formulated as a mixed-integer nonlinear program and two-phase hybrid solution approaches employing two different heuristics are developed.
\end{abstract}

\section{INTRODUCTION}

Wavelength division multiplexing (WDM) is an important technique used to take advantage of the enormous bandwidth in fiber optics [1]. An optical network consists of an interconnection of stations, switches and other devices using optical fiber. Access stations, or simply stations, in this network are able to inject (receive) traffic into (from) the network. A connection in an optical network is set up on a lightpath. A lightpath between two stations is an available wavelength on a series of fiber links from source to destination such that the route is simple (i.e., free of cycles) and the signal remains in the optical domain (i.e., it is not converted to electronics).

The throughput version of the Routing and Wavelength Assignment (RWA) problem [2] involves selecting the best route (path) and wavelength for each connection (for a given demand matrix) such that the maximum number of connections are established. Previous studies have solved many variations of this problem under the assumption of perfect conditions regarding the power of a signal. In this paper, we investigate the problem while accounting for the power degradation of a routed signal due to the non-ideal behavior of optical components such as multiplexers, demultiplexers, taps, and fiber links.

This paper is organized as follows. In Section II, the all-optical network architecture is introduced. Section III gives the mathematical formulation of the problem. In Section IV, a two-phase solution approach to this problem is presented along with numerical examples. Finally, Conclusion is covered in Section V.

\section{NETwORK AND NODE ARCHITECTURE}

A pair of unidirectional fibers connect nodes in the network. Long fiber links may be interspersed with inline amplifiers. Traffic on a WDM channel can be transferred from one link to another at a switching node. A switching node contains components such as taps, input/output amplifiers, multiplexers, etc., and wavelength-routing switches (WRS). Figure 1 shows a typical switching node in an optical network. This $4 \times 4$ switch contains only one station. We distinguish between switches and stations, despite the fact that they may be implemented as one unit. The number of input/output ports is always a power of 2 (e.g., a $3 \times 3$ switch is implemented as a $4 \times 4$ switch.). Three wavelengths $\lambda_{1}, \lambda_{2}, \lambda_{3}$ are used on the links. A signal entering a switch encounters various components which contribute to loss/gain. The switch power loss for a $D_{i} \times D_{i}$ switch is given by $2\left\lceil\log _{2}\left(D_{i}\right)\right\rceil L_{s}+4 L_{w}$, where $D_{i}$ is the node degree, $L_{s}$ is the insertion loss, and $L_{w}$ is the coupling loss [3]. In order to establish a connection in this network, we not only need a free wavelength on a route, but also need enough power on the signal for it to reach the receiving station. In this paper, we assume that optical amplifiers are preplaced on individual links by the network engineer. Note that we do not consider, at present, the bit-error rate of the channels or the effects of dispersion, crosstalk and fiber nonlinearities.

\section{Problem Formulation}

In this section, the RWA problem for optical networks with power considerations (henceforth referred to as RWA-P) is formulated as a mixed-integer non-linear program (MINLP).

\section{A. Amplifier Gain Model}

Optical amplification is assumed to be accomplished using Erbium-Doped Fiber Amplifiers (EDFAs) [4]. One major issue which contributes to the complexity of the RWA-P problem is the fact that the gain of an EDFA 
amplifier is a function of the total input power over all wavelengths; hence amplification gain is non-constant.

The gain available at an optical amplifier is given by the following function:

$$
G\left(P_{i n}\right)=\min \left\{G_{\max },\left(P_{\max }-P_{i n}\right)\right\}
$$

where $P_{i n}$ is the total input power, $P_{\max }$ is the maximum amplifier power, and $G_{\max }$ is the small-signal gain. Note that we assume equal gain for all connections entering a specific amplifier, regardless of their individual power levels. More sophisticated gain models may be substituted without affecting the formulation.

\section{B. Device Parameters}

Table 1 shows the values of different device parameters used in this study. These parameters are as follows.

- $p_{\text {sen }}=$ minimum power required on a wavelength for detection in $\mathrm{dBm}$ This represents both the receiver and amplifier sensitivity levels, which we assume to be equal.

- $P_{\max }=$ maximum power available from an amplifier in $\mathrm{mW}$.

- $P_{m a x}^{\text {max }}=\max$ transmission power in $\mathrm{mW}$.

- $G_{\max }=\max ($ small-signal) amplifier gain in $\mathrm{dB}$.

- $\alpha=$ signal attenuation on a fiber in $\mathrm{dB} / \mathrm{km}$.

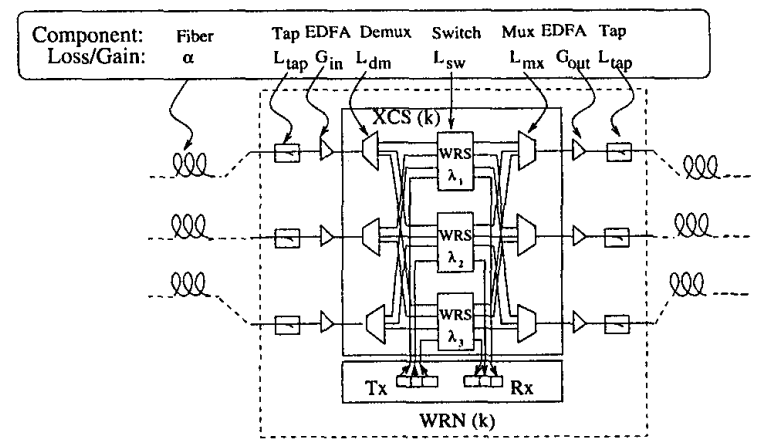

Figure 1: A Switching Node.

\section{Notation}

We define the notation used in the problem formulation. The inputs to the problem are:

- Network topology represented as a directed graph $G(V, E, N, M)$, where $N$ is the number of stations, $M$ is the number of switches, $L=|E|$ the number of unidirectional links, and $|V|=(N+M)$.

\begin{tabular}{||l|l||}
\hline Parameter & Value \\
\hline Multiplexer loss $\left(L_{m x}\right)$ & $4 \mathrm{~dB}$ \\
\hline Demultiplexer loss $\left(L_{d m}\right)$ & $4 \mathrm{~dB}$ \\
\hline Switch element insertion loss $\left(L_{s}\right)$ & $1 \mathrm{~dB}$ \\
\hline Waveguide/fiber coupling loss $\left(L_{w}\right)$ & $1 \mathrm{~dB}$ \\
\hline Tap loss $\left(L_{t a p}\right)$ & $1 \mathrm{~dB}$ \\
\hline Fiber loss $(\alpha)$ & $0.2 \mathrm{~dB} / \mathrm{km}$ \\
\hline $\begin{array}{l}\text { Maximum amplifier output power } \\
\left(P_{\text {max }}\right)\end{array}$ & $1 \mathrm{~mW}(0 \mathrm{dBm})$ \\
\hline $\begin{array}{l}\text { Maximum transmitter power } \\
\left(P_{\text {max }}^{x m i t}\right)\end{array}$ & $1 \mathrm{~mW}(0 \mathrm{dBm})$ \\
\hline $\begin{array}{l}\text { Minimum signal power at amplifier } \\
\text { and receiver }\left(p_{\text {sen }}\right)\end{array}$ & $-30 \mathrm{dBm}$ \\
\hline $\begin{array}{l}\text { Maximum small-signal } \\
\text { gain of inline amplifier }\left(G_{m a x}\right)\end{array}$ & $20 \mathrm{~dB}$ \\
\hline Input EDFA Gain $\left(G_{i n}\right)$ & $12 \mathrm{~dB}$ \\
\hline Output EDFA Gain $\left(G_{o u t}\right)$ & $12 \mathrm{~dB}$ \\
\hline
\end{tabular}

Table 1

System parameters and values used in this study.

- Demand matrix $T_{(N \times N)}$. Matrix $T$ specifies call demands between node pairs. $T_{s, d}=$ the number of all-optical lightpaths that are desired from node $s$ to node $d$.

In the formulation, stations are identified by the indices $1,2, \ldots, N$. Switches are identified by the indices $(N+$ $1),(N+2), \ldots,(N+M)$.

We are also given the following

- $W=$ number of wavelengths on a link.

- $s_{l}=$ source of link $l, 1 \leq s_{l} \leq(N+M)$.

- $d_{l}=$ destination of link $l, 1 \leq d_{l} \leq(N+M)$.

- $L_{l}=$ length of link $l$ in $\mathrm{km}$.

- $\nabla_{l}=$ number of devices on link $l$.

- $\eta_{l, k}=$ the length of the fiber (component) $k$ on link $l$ in $\mathrm{km}, 1 \leq k \leq\left(2 \nabla_{l}+1\right)$. See subsection 2 for more information.

- $D_{i}=$ in-degree $=$ out-degree of switch $i$.

- $O U T_{i}=$ the set of links with switch $i$ as source.

- $I N_{i}=$ the set of links with switch $i$ as destination.

- $R_{s, d, l}=$ the routing-table entry for a connection pair $(s, d)$. Every station pair $(s, d)$ has one route (e.g the shortest path). If link $l$ in the network is on the route from station $s$ to station $d$ then $R_{s, d, l}=1 ; R_{s, d, l}=0$, otherwise. 
- $\xi_{s, d, l}=$ link following link $l$ on path from $s$ to $d$.

We require the solver to solve for the variables grouped into three categories: routing, link, and node variables. These categories are described below.

\section{1) Routing variables}

Since the route of a connection is known beforehand, only one variable per connection is needed: $\Lambda_{s, d, c}$, the connection wavelength (binary) variable. $\Lambda_{s, d, c}=1$, if one of the lightpaths used in the established connections from station $s$ to $d$ uses wavelength $c ; \Lambda_{s, d, c}=0$, otherwise.

\section{2) Link variables}

This section introduces the variables indexed by links. We use the convention that capital letter variables refer to aggregate power levels on the link and are measured in $\mathrm{mW}$. Lowercase variables refer to the power levels per-wavelength and are measured in $\mathrm{dBm}$. Every link in the network is partitioned into different components. A component is a device or a fiber segment which contributes to the signal power level either by a loss or a gain. An example of a component is an amplifier. If the link does not contain any devices, then the link has only one component, the fiber segment itself. In general, a link $l$ which has $\nabla_{l}$ devices, has $\left(2 \nabla_{l}+1\right)$ components. The reason for introducing many variables for a link is that we want to make sure that the power levels of signals are within acceptable levels at the beginning and at the end of each component defined on every link in the network. We only make use of the following devices: inline amplifiers, input amplifiers, output amplifiers, input taps, output taps, demultiplexers, and multiplexers. Other devices can be easily added given their corresponding gain/loss function.

The variable $p_{c, s, d, l, x}^{b e g}\left(p_{c, s, d, l, x}^{e n d}\right)$ represents the power on wavelength $c$ for connection $(s, d)$ at the beginning (end) of component $x$ on link $l$ and it should not be below $p_{\text {sen }}$ nor above $P_{\max }$. It is also important to ensure that the aggregate power at the beginning $\left(P_{l, x}^{b e g}\right)$ and at the end $\left(P_{l, x}^{e n d}\right)$ of each component $x$ be within the valid power levels mentioned above. Figure 2 shows some of the power-level variables for components 10 through 17 on link $y$. Only a small portion of the link and only variables pertaining to wavelength 1 are shown.

\section{3) Node Variables}

Consider switch, $i,(N+1) \leq i \leq(N+M)$ and link $l \in O U T_{i}$. The real variable $p_{c, s, d, i, l}^{\text {switch }}$ out denotes the power on wavelength $c$ at the output of switch $i$ (attached to link $l$ ) for connection $(s, d)$ in $\mathrm{dBm}$. Also consider station $s, 1 \leq s \leq N$. The transmission power of station $s$ on wavelength $c$ for connection $(s, d)$ in $\mathrm{dBm}$ is given by the real variable $p_{c, s, d}^{x m i t}$.

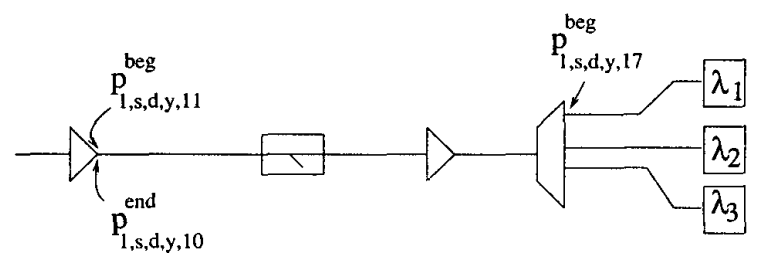

Figure 2: Some of the power-level variables for components 10 through 17 on link $y$.

\section{4) Useful Functions}

The following functions are used in the formulation to convert between the $\mathrm{mW}$ (regular) and $\mathrm{dBm}(\log )$ scales.

$\operatorname{ToDB}(\xi)=10 \log _{10}(\xi)$

$\operatorname{ToMW}(\xi)=10^{\xi / 10}$

\section{Constraints}

The constraints are divided into two groups. We discuss the routing constraints and the power constraints.

\section{1) Routing Constraints}

The following constraint ensures that a lightpath is established only if the user requests it.

$$
\sum_{c=1}^{W} \Lambda_{s, d, c} \leq T_{s, d} \quad 1 \leq s \leq N, 1 \leq d \leq N
$$

Any link should not be used to route more than $W$ connections (capacity constraint).

$$
\sum_{s=1}^{N} \sum_{d=1}^{N} \sum_{c=1}^{W} \Lambda_{s, d, c} * R_{s, d, l} \leq W \quad 1 \leq l \leq L .
$$

Two lightpaths which share the same physical link $l$, must use different wavelengths.

$$
\sum_{s=1}^{N} \sum_{d=1}^{N} \Lambda_{s, d, c} * R_{s, d, l} \leq 1 \quad 1 \leq l \leq L, 1 \leq c \leq W .
$$

Finally, the wavelength (color) variable is binary.

$$
\Lambda_{s, d, c} \in\{0,1\} \text {. }
$$

\section{2) Power Constraints}

The transmission power on wavelength $c$ from station $s$ to station $d$ should be within valid power levels.

$$
p_{s e n} \leq p_{c, s, d}^{x m i t} \leq \operatorname{ToDB}\left(P_{\max }^{x m i t}\right)
$$

For switch $i$ and link $l$, where $l \in O U T_{i}$, we have

$$
p_{c, s, d, i, l}^{s w i t c h \_o u t}=p_{c, s, d, \pi,\left(2 \nabla_{n}+1\right)}^{\text {end }}-\operatorname{LOSS}_{i} * \Lambda_{s, d, c}
$$


where $L O S S_{i}$ is the switch loss $\left(L_{s w}\right)$ of switch $i$ (see Section II) and $\xi_{s, d, \pi}=l$. The power on wavelength $c$ at the beginning of the first component depends on whether the source of link $l$ is a station or a switch.

$$
\begin{gathered}
p_{c, s, d, l, 1}^{b e g}=p_{c, s, d}^{x m i t} \quad 1 \leq s_{l} \leq N \\
p_{c, s, d, l, 1}^{\text {beg }}=p_{c, s, d, i, l}^{\text {switch out }} \quad(N+1) \leq i=s_{l} \leq(N+M) .
\end{gathered}
$$

The power on wavelength $c$ at the beginning of component $k, k \neq 1$, on link $l$ is simply

$$
p_{c, s, d, l, k}^{b e g}=p_{c, s, d, l, k-1}^{e n d}
$$

The total power in $\mathrm{mW}$ at the beginning of component $k$ on link $l$ is defined to be

$$
P_{l, k}^{b e g}=\sum_{s \in N} \sum_{d \in N} \sum_{c \in W} T o M W\left(p_{c, s, d, l, k}^{b e g}\right) * \Lambda_{s, d, c}
$$

Defined similarly, the total power in $\mathrm{mW}$ at the end of component $k$ on link $l$ is

$$
P_{l, k}^{e n d}=\sum_{s \in N} \sum_{d \in N} \sum_{c \in W} T o M W\left(p_{c, s, d, l, k}^{e n d}\right) * \Lambda_{s, d, c}
$$

Let us define the function $\Upsilon_{l, k}\left(P_{i n}\right)$ to be the gain/loss contribution of component $k$ on link $l$.

$$
\Upsilon_{l, k}\left(P_{i n}\right)= \begin{cases}-L_{t a p} & \text {-input tap } \\ -L_{m x} & \text {-multiplexer } \\ -L_{d m} & \text {-demultiplexer } \\ -\alpha * \eta_{l, k} & \text {-fiber link } \\ G\left(P_{i n}\right) & \text {-inline amplifier } \\ G\left(P_{i n}\right) & \text {-input amplifier } \\ G\left(P_{i n}\right) & \text {-output amplifier }\end{cases}
$$

where $P_{i n}$ is the total aggregate power of all the connections which pass through the component $k$ (i.e., $\left.P_{i n}=P_{l, k}^{b e g}\right)$ and $G_{i n}\left(G_{o u t}\right)$ replaces $G_{\max }$ in the gain function in the case of input (output) EDFA amplifier. Recall that $\eta_{l, k}$ is the length of the fiber segment (component) $k$ of link $l$. In this study, all components except the fiber segments have zero length. Given the value $\Upsilon_{l, k}\left(P_{i n}\right)$, we have the following.

$$
p_{c, s, d, l, k}^{e n d}=p_{c, s, d, l, k}^{b e g}+\Upsilon_{l, k}\left(P_{i n}\right)
$$

The individual power levels at the beginning of every component should be within acceptable power levels.

$$
p_{s e n} \leq p_{c, s, d, l, x}^{b e g} * \Lambda_{s, d, c} \leq \operatorname{ToD} B\left(P_{\max }\right)
$$

Similarly, for the power at the end of component,

$$
p_{s e n} \leq p_{c, s, d, l, x}^{e n d} * \Lambda_{s, d, c} \leq \operatorname{ToD} B\left(P_{\max }\right)
$$

The total power at the beginning and at the end of every component $x, x \neq 1$ is the sum of all individual powers after appropriate scale changes and should be within acceptable power levels.

$$
\begin{aligned}
& \operatorname{ToMW}\left(p_{s e n}\right) \leq P_{l, k}^{b e g} \leq P_{\max } \\
& \operatorname{ToMW}\left(p_{s e n}\right) \leq P_{l, k}^{e n d} \leq P_{\max }
\end{aligned}
$$

\section{E. Objective Function}

The objective is simply to maximize the total number of connections successfully routed in network.

$$
\text { Maximize } Z=\sum_{s=1}^{N} \sum_{d=1}^{N} \sum_{c=1}^{W} \Lambda_{s, d, c}
$$

\section{F. Reasons for Nonlinearity}

The first reason for nonlinearity is the non-linear gain model in Eqn. 1. The other reason is the presence of product terms of the individual power level and the routability of a connection (an integer variable) in constraints $11,12,14$, and 15 .

\section{Two-Phase Solution Approach}

Clearly, the formulation given in the previous section is a very difficult problem to solve. We introduce a two-phase approach to handle the complexity of the problem. We divide the problem into two sub-problems. The first sub-problem is the classic RWA problem, neglecting the power constraints, which can be solved using an ILP solver (or any graph coloring-based method). The output of the ILP RWA solution is fed into a Genetic Algorithm (GA)module or alternatively, to a heuristic we call Smallest-Gain First (SGF). The formulation of the RWA is along the lines in [2] and is not shown here. In order to cut down on the enormous search space, a sub-optimal ILP solution (without adaptive routing) is considered. A precomputed set of $k$-shortest paths is used to select routes for every node pair in the demand matrix. The SGF heuristic sorts the connections in increasing order according to Eqn. (19) which finds the total amount of gain/loss that connection $i$ encounters assuming that no other connections are present in the network. Given that sorted list, SGF tries to establish connections one by one in that order. A connection is rejected if the power of an already established connection drops due to a saturated amplifier.

$$
g_{i}=\sum_{l \in p a t h[i]}\left(L O S S_{d_{l}}+\sum_{c \in l} \Upsilon_{l, c}(0.0)\right)
$$

The genetic algorithm, on the other hand, starts with a set of random solutions. These solutions are evolved over 
time in a way that better solutions in the enormous search space are discovered. For more information on the GA solution approach refer to [5].

\section{A. Numerical Examples}

The two heuristics were run on the sample optical network given in Fig. 3. All experiments were conducted on a lightly-loaded $400 \mathrm{MHz}$ PC running Windows NT 4.0. Table 2 shows the results obtained by varying the number of wavelengths, given the demand matrix (20).

$$
\text { Demand }=\left[\begin{array}{llllll}
0 & 3 & 2 & 0 & 2 & 1 \\
0 & 0 & 3 & 0 & 0 & 0 \\
0 & 1 & 0 & 1 & 0 & 3 \\
1 & 3 & 2 & 0 & 3 & 2 \\
0 & 0 & 0 & 3 & 0 & 0 \\
1 & 2 & 3 & 1 & 2 & 0
\end{array}\right]
$$

In each case, the first column gives the number of connections generated by the linear solver (which serves as an upper bound on the number of connections for the heuristics) and the time (in seconds) it took to solve the program. In the second column, the number of established connections using the SGF heuristic is given. Finally, the last column lists the result obtained from the genetic algorithm (GA). We find that our genetic algorithm based heuristic performs better than the SGF heuristic. For small $W$, the results obtained from our GA based heuristic achieves the upper bound on the number of connections. Table 3 shows the results obtained when all other variables are kept fixed except the traffic matrix. We find that the GA did not help much in the two extreme cases: Sparse and Max. The other two cases show a justification for spending additional time by using the genetic algorithm.

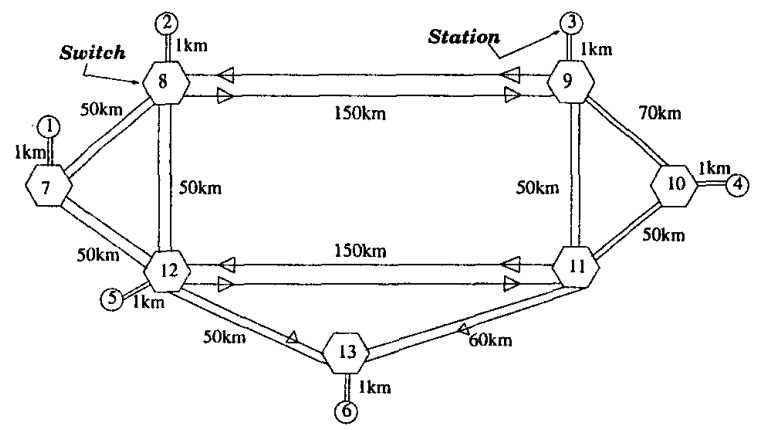

Figure 3: An example of a switched all-optical network.

\section{CONCLUSION}

In this paper, we introduced the problem of routing and wavelength assignment in optical networks while

\begin{tabular}{||l|l|l|l||}
\hline W & LP & SGF & GA \\
\hline 6 & $28 / 213 s$ & $27 / 0.1 s$ & $28 / 521 s$ \\
\hline 7 & $31 / 289 s$ & $26 / 0.1 s$ & $31 / 633 s$ \\
\hline 8 & $33 / 360 s$ & $27 / 0.1 s$ & $32 / 721 s$ \\
\hline 9 & $35 / 631 s$ & $28 / 0.1 s$ & $33 / 805 s$ \\
\hline 10 & $37 / 926 s$ & $27 / 0.1 s$ & $33 / 933 s$ \\
\hline 11 & $38 / 1214 s$ & $27 / 0.1 s$ & $34 / 997 s$ \\
\hline
\end{tabular}

Table 2

Traffic matrix in (20) applied to the mesh network in Fig. 3.

\begin{tabular}{||l|l|l|l||}
\hline Demand Matrix & LP & SGF & GA \\
\hline Uniform $[1, W]$ & $33 / 360 s$ & $27 / 0.1 s$ & $32 / 720 s$ \\
\hline Constant: $\frac{W}{2}$ & $48 / 33 s$ & $35 / 0.1 s$ & $44 / 378 s$ \\
\hline Max: $W$ & $48 / 120 s$ & $44 / 0.1 s$ & $44 / 478 s$ \\
\hline Sparse: Uniform $[0,1]$ & $14 / 40 s$ & $13 / 0.1 s$ & $14 / 15 s$ \\
\hline
\end{tabular}

Table 3

Using different traffic demand for the mesh network in Fig. 3. considering power constraints. We formulated the problem as a mixed-integer nonlinear program. Then to overcome the problems due to nonlinear constraints, we devised a two-phase hybrid solution approach employing either a genetic or a greedy algorithm. Our GA-based approach was compared to another hybrid solution approach using the Smallest-Gain First (SGF) heuristic. The results indicate that our GA-based approach performs well for a wide choice of parameters. The additional time spent searching using the genetic algorithm usually results in a better solution.

\section{REFERENCES}

[1] B. Mukherjee, Optical Communication Networks. New York : McGraw-Hill, 1997.

[2] R. Ramaswami and K. N. Sivarajan, "Routing and wavelength assignment in all-optical networks," IEEE/ACM Trans. on Networking, vol. 3, 1995.

[3] R. A. Spanke, "Architectures for guided-wave optical space switching systems," IEEE Communications Magazine, vol. 25, May 1987.

[4] E. Desurvire, Erbium-Doped Fiber Amplifiers: Principles and Appl. New York, NY: Wiley, 1994.

[5] M. Ali, B. Ramamurthy, and J. S. Deogun, "Routing algorithms for all-optical networks with power considerations: The unicast case," in ICCCN, 1999. 